Association for Information Systems

AIS Electronic Library (AISeL)

Wirtschaftsinformatik Proceedings 1999

Wirtschaftsinformatik

February 1999

\title{
Wie lernt der Computer den Menschen kennen? Bestandsaufnahme und Experimente zur Benutzermodellierung in der Wirtschaftsinformatik
}

Peter Mertens

Universität Erlangen-Nürnberg, mertens@wiso.uni-erlangen.de

Michael Höhl

Universität Erlangen-Nürnberg, hoehl@wiso.uni-erlangen.de

Follow this and additional works at: http://aisel.aisnet.org/wi1999

\section{Recommended Citation}

Mertens, Peter and Höhl, Michael, "Wie lernt der Computer den Menschen kennen? Bestandsaufnahme und Experimente zur Benutzermodellierung in der Wirtschaftsinformatik" (1999). Wirtschaftsinformatik Proceedings 1999. 3.

http://aisel.aisnet.org/wi1999/3

This material is brought to you by the Wirtschaftsinformatik at AIS Electronic Library (AISeL). It has been accepted for inclusion in Wirtschaftsinformatik Proceedings 1999 by an authorized administrator of AIS Electronic Library (AISeL). For more information, please contact elibrary@aisnet.org. 


\section{Wie lernt der Computer den Menschen kennen? Bestandsaufnahme und Experimente zur Benutzermodellierung in der Wirtschaftsinformatik}

Peter Mertens

Universität Erlangen-Nürnberg (mertens@ wiso.uni-erlangen.de)

Michael Höhl

Universität Erlangen-Nürnberg (hoehl@wiso.uni-erlangen.de)

\section{Inhalt}

1 Einleitung

2 Benutzermodellierung in der Wirtschaftsinformatik

2.1 Cui bono?

2.2 Rahmenplan zur Benutzermodellierung

3 Bestandsaufnahme der Benutzermodellierung

3.1 Informationen für Fach- und Führungskräfte

3.2 Verkauf

3.3 Schulung und Beratung

3.4 Mensch-Aufgabe-Zuordnung

4 Eigene Experimente

4.1 Informationen für Fach- und Führungskräfte

4.1.1 Managementinformationen aus dem Internet

4.1.2 Integration von Controlling- und Marktforschungsdaten in einem Expertisesystem

4.1.3 Unternehmensreport II

4.1.4 Client-Oriented Branch Reporting and Analysis System

4.2 Verkauf

4.2.1 Infobroker

4.2.2 Electronic Personal Online Shopper

4.2.3 Produktberatungskomponente

4.2.4 Software Consultant

4.3 Schulung und Beratung

4.3.1 Adaptives Multimediales Präsentationssystem

4.3.2 Stadtinformationssystem

5 Hindernisse und Gefahren

6 Resümee und Ausblick 


\begin{abstract}
Benutzermodelle ${ }^{1}$ sind ein wichtiger Baustein auf dem Weg zu einer sinnhaften Vollautomation der Informationsverarbeitung (IV). Indem sie dafür sorgen, dass ,sich Computer und Mensch gut verstehen“, erhöhen sie die Akzeptanz von Anwendungssystemen. Darüber hinaus helfen sie, Informationen im Internet $\mathrm{zu}$ suchen, zu filtern und individuell zu präsentieren. Dennoch zögern Praktiker, Benutzermodellierungskomponenten in Anwendungssysteme zu integrieren. Ein „Blick in die eigene Werkstatt“ skizziert pragmatische Ansätze, die geeignet sein mögen, „Berührungsängste“ mit einem komplexen Forschungsfeld abzubauen.
\end{abstract}

\title{
1 Einleitung
}

Mit diesem Beitrag wollen wir an das von Mitautor Mertens auf der WI `95 postulierte Langfristziel der Wirtschaftsinformatik, die sinnhafte Vollautomation, anknüpfen (Mertens 1995a, S. 48-50). Das Attribut „sinnhaft“ meint, dass ein Automationsschritt von der Allgemeinheit nach einer Lernfrist akzeptiert wird und sich allenfalls Nostalgiker und Sonderlinge nach der personellen Lösung zurücksehnen. Abbildung 1 skizziert die Wege zur Vollautomation.

Ein wichtiges Unterziel ist es, den Computer menschenähnlicher zu machen (menschenähnliche IV). Dieses Unterziel kann man damit motivieren, dass der Mensch ein aus langen darwinistischen Prozessen hervorgegangener guter Informationsverarbeiter und damit als Vorbild für die maschinelle IV über weite Strecken geeignet ist.

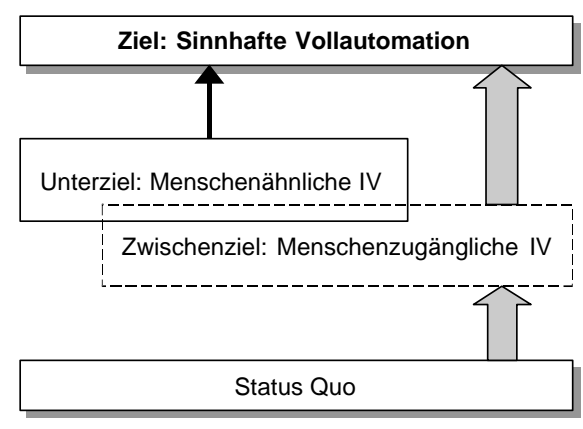

Abbildung 1: Wege zur Vollautomation

Da die Vollautomation auf vielen Gebieten lange auf sich warten lassen wird, ist als „Etappe“ anzusteuern, dass möglichst breite Kreise der Bevölkerung IuK-Systeme im teilautomatisch/interaktiven Betrieb leicht bedienen können und dann gerne nutzen (menschenzugängliche IV). Die hierfür notwendigen Aus- und Weiterbildungsmaßnahmen müssen sich in Grenzen halten. Diese Prämisse folgt aus der Notwendigkeit, relative Laien nicht durch IV-Systeme auszumanövrieren,

1 Unsere Leserinnen bitten wir um Verständnis, dass wir nicht den schwerfälligen Begriff „Benutzerinnen- und Benutzermodellierung“ verwenden. 
ebenso wie aus volkswirtschaftlichen Zwängen, was die Relation zwischen Schulungs- und produktiven Lebensphasen betrifft.

Menschenzugängliche IV ist nur zu erreichen, wenn sich der Computer dem Menschen anpasst und nicht umgekehrt. Da es ,den Menschen“ aber nicht gibt, ist es notwendig, Systeme $\mathrm{zu}$ erstellen, welche individuelle Fähigkeiten, Vorkenntnisse und Ziele berücksichtigen, indem sie Benutzermodelle aufbauen. In einer pragmatischen Abgrenzung verstehen wir darunter Mechanismen, die es dem Computer erlauben, sich möglichst gut auf den Menschen einzustellen.

Während die theoretische Fundierung der Benutzermodellierung in der Wissenschaft schon weit fortgeschritten ist (Verweise auf Forschungsprojekte finden sich beispielsweise auf der Internet-Seite von Wahlster $)^{2}$, scheinen Praktiker noch vor entsprechenden Implementierungen, speziell im Internet, zurückzuschrecken (s. Kap. 3). Die Gründe für diese Lücke zwischen Wissenschaft und Praxis sind vielschichtig: Datenschutz und -sicherheit, hoher Realisierungsaufwand und Einflussgrößen wie Rechenaufwand und Speicherbedarf tragen zur Verunsicherung bei. Das große Dilemma der Modellierer liegt jedoch in der Herausforderung, Benutzer treffend zu taxieren, ohne sie in umfangreiche, zusätzliche Dialoge (Interviews) zu verwickeln.

\section{Benutzermodellierung in der Wirtschaftsinformatik}

\subsection{Cui bono?}

1. Es ist eine wesentliche Aufgabe der Benutzermodellierung, Informationen für Fach- und Führungskräfte entsprechend den individuellen Anforderungen des Anwenders zu suchen, zu filtern und zu präsentieren.

2. Nicolas Negroponte schreibt, wir befänden uns auf dem Weg vom Informations- ins Postinformationszeitalter (Negroponte 1995, S. 201). Letzteres zeichne sich durch das so genannte „Einpersonenpublikum“ aus, d. h., Massenkommunikation werde durch Individualkommunikation ersetzt. Dieser Trend spiegelt sich auch in Schlagworten des Marketing wider, wie z. B. „One-to-One“, „Segment-of-One“ und „Relationship“ (vgl. Peppers 1993; Kotler 1997). Neben der Kommunikationspolitik versucht man, Produkte auf Massenmärkten an spezifischen Bedürfnissen auszurichten (Mass Customization; Mertens 1995b).

So verwundert es nicht, dass verschiedene Studien der letzten Monate betonen, der Fokus der nächsten Jahre müsse auf einer radikalen Orientierung am Kunden liegen (vgl. O. V. 1998a). Im Database Marketing nutzt man

2 Aus Platzgründen findet sich die Liste der im Beitrag zitierten WWW-Adressen im Internet unter http://www.wil.uni-erlangen.de/ hhl/BM.html. 
beispielsweise die Verkaufsdaten der Kunden, um individuell aktuellere oder zusätzliche Produkte anzubieten (Cross-Selling, Up-Selling; vgl. Link 1993). Etwas weiter führen Ideen, Kunden „lebenslang“ zu betreuen. So könnte man in einem Benutzermodell Entwicklungspfade abspeichern, um zukünftige Bedarfe zu antizipieren. Ein Autohändler etwa würde seiner langjährigen Kundin nach bestandenem Examen einen schicken Sportwagen empfehlen, da er davon ausgeht, dass ein teueres Fahrzeug nun erschwinglich ist. Weiß der Verkäufer aber, dass er eine junge Mutter vor sich hat, so würde er möglicherweise ein stärkeres Sicherheitsbewusstsein in seiner Offerte berücksichtigen und darüber hinaus ein geräumigeres Fahrzeug favorisieren.

3. Schulungs- und Beratungssysteme sind eine klassische Domäne der Benutzermodellierung. In Anwendungen des Computer-based Training und in Intelligenten Tutoriellen Systemen hinterlegt man didaktische Konzepte, um auf die Lernfortschritte der Probanden durch angepasste Lehrinhalte zu reagieren (Langer 1995). Da die Kenntnisse der Teilnehmer explizit abgefragt werden können, lassen sich effizient Benutzermodelle erstellen, die z. B. auch mediale Präferenzen, wie Texte, Grafiken und Videos, enthalten. Beratungssysteme, die Ratschläge für Investitionen an der Börse erteilen, können anlegerspezifische Parameter wie die Risikoaversion speichern, um entsprechende Portfolios zusammenzustellen.

4. Software für die Mensch-Aufgabe-Zuordnung sollte über Modelle der Rollen, Fähigkeiten und aktuellen Auslastungen von Mitarbeitern verfügen. In Workflow-Management-Systemen (WMS) ermöglicht dies eine dynamische und effiziente Arbeitsbevorratung, da sichergestellt ist, dass Vorgänge direkt zu den Mitarbeitern mit dem erforderlichen Know-how geleitet werden (Oberweis 1996). Durchlaufzeiten lassen sich auf diese Weise verkürzen. Gekoppelt an betriebswirtschaftliche Standardsoftware können die Benutzermodelle aus dem WMS auch der Parametrierung des Programms dienen (Becker 1998, S. 320).

Eine reizvolle Variante ist es, die mitarbeiterspezifischen Daten eines solchen WMS wiederum als Grundlage für die Informationssuche von Fachund Führungskräften einzusetzen. Das Wissensmanagement-Tool Answer Garden listet z. B. Experten auf, die über das relevante Know-how zur Lösung eines gegebenen Problems verfügen (Ackerman 1994, S. 31). Die besondere Herausforderung liegt in diesem Fall in der Wissensakquisition, da nicht nur die Experten zu identifizieren sind, sondern beurteilt werden muss, wer ehrliche Auskünfte erteilt oder aber sein Wissen zurückhält.

\subsection{Rahmenplan zur Benutzermodellierung}

Adaptiven Systemen liegen Benutzermodelle zur Selektion und zur Präsentation zugrunde (s. Abb. 2). Benutzermodelle zur Selektion binden Präferenzen des Anwenders in die Suche ein, um Informationen und Produkte zu finden und zu bewerten. Solche zur Präsentation steuern den Dialogablauf entsprechend dem Wissensstand des Anwenders und legen fest, in welcher Form Inhalte aufbereitet 
werden. Diese können auf der einen Seite die Domäne selbst - also problemspezifisches Wissen - betreffen, zum anderen aber auch den Umgang mit dem System.

\begin{tabular}{|c|c|c|c|}
\hline Merkmale & \multicolumn{3}{|c|}{ Ausprägungen } \\
\hline \multirow[t]{2}{*}{ Zweck } & \multirow[t]{2}{*}{ Selektion } & \multicolumn{2}{|c|}{ Präsentation } \\
\hline & & Domäne & System \\
\hline \multirow[t]{2}{*}{ Gegenstand } & \multicolumn{2}{|l|}{ Empfänger } & \multirow[t]{2}{*}{ Bediener } \\
\hline & Kunde $\quad$ Rolle $\quad$ Organis. & Gruppe & \\
\hline Individualisierung & individuell & \multicolumn{2}{|c|}{ differenzierend } \\
\hline Art der Informationen & weiche Informationen & \multicolumn{2}{|c|}{ harte Fakten } \\
\hline Veränderbarkeit & statisch & \multicolumn{2}{|c|}{ dynamisch } \\
\hline \multirow[t]{2}{*}{ Gewinnung } & \multirow[t]{2}{*}{ implizit } & \multicolumn{2}{|c|}{ explizit } \\
\hline & & ex ante & ex post \\
\hline Einsichtigkeit & transparent & \multicolumn{2}{|c|}{ intransparent } \\
\hline Gültigkeit & langfristig & \multicolumn{2}{|c|}{ kurzfristig } \\
\hline Wissensakquisition & personell & \multicolumn{2}{|c|}{ lernend } \\
\hline
\end{tabular}

Abbildung 2: Merkmale von Benutzermodellen

Bezüglich des Gegenstands der Benutzermodellierung ist zu unterscheiden, ob der Bediener allein die Determinanten der zu lösenden Aufgabe bestimmt oder ob er - gewissermaßen als Intermediär - den oder die Empfänger vertritt (Wahlster und Kobsa sprechen von einem „Agent Model“; Wahlster 1989, S. 7). Im zweiten Fall kann es sich um einen Kunden, eine Gruppe von Personen oder abstrakte Gebilde wie die Rolle eines Mitarbeiters oder eine Organisation handeln. Möchte sich ein Steuerberater beispielsweise über Software für seine Kanzlei informieren, so sind seine persönlichen Präferenzen weniger wichtig als die objektiven Anforderungen seines Betriebes, d. h., die Zwänge einer Rolle oder das Umfeld eines Unternehmens dominieren persönliche Präferenzen.

In diesem Zusammenhang ist zwischen einem subjektiven und einem objektiven Informationsbedarf abzuwägen: So kann eine Aufgabe bzw. Rolle zwar objektiv bestimmte Informationen erfordern. Es ist aber denkbar, dass ein Manager unkonventionelle Problemlösungswege beschreitet, sein - subjektiver - Informationsbedarf ist dann ein anderer.

Entscheidet eine Gruppe gemeinsam über die Alternativen einer Problemlösung, so ist zu klären, nach welchen Strategien die Präferenzen der Gruppenmitglieder zu berücksichtigen sind (z. B. Mittelwerte, Restriktionen).

Nach dem Grad der Individualisierung lassen sich einerseits Benutzermodelle finden, die sich individuell an die Bedürfnisse eines Anwenders oder Empfängers anpassen. Andererseits versucht man häufig, die Komplexität solcher Systeme mit Hilfe von Stereotypen einzuschränken (differenzierend), d. h., man ordnet dem Benutzer „ein Bündel von Eigenschaften [zu] ..., wenn bestimmte 
auslösende Informationen, die sogenannten Trigger, bekannt werden.“ (Bodendorf 1992, S. 241).

Das Content-based Filtering, eine Variante dieser differenzierenden Systeme, beschreibt Anwender mit Schlüsselbegriffen der bereits abgerufenen Informationen. Ausgefeilte Retrieval-Software bewertet relevante Fundstellen nach der Häufigkeit der Begriffe, die auch im Benutzerprofil enthalten sind, und gewichtet nach deren Position in den Dokumenten. Die Qualität der Suchergebnisse bleibt aber unberücksichtigt.

Das Social oder Collaborative Filtering (auch „Recommender Systems“; Resnick 1997; Mertens 1997) versucht verlässlichere Ratschläge zu erteilen, indem es Benutzer in „Communities“ einordnet und Lösungen empfiehlt, die anderen Mitgliedern dieser Interessengemeinschaften weiterhalfen (Oard 1997, S. 149-150). Da Dokumente in Recommender Systems durch Annotationen der Leser repräsentiert werden, kann man erst Vorschläge erzeugen, wenn genügend Datensätze gespeichert sind. Fehlen Hinweise früherer Rechercheure, so sinkt die Motivation, ein solches System zu benutzen („Henne-Ei-Problem“). Man diskutiert deshalb über Anreize, die potenzielle Erstanwender anregen sollen, Collaborative-Filtering-Software mit Startwerten zu versorgen.

Implementierungen, die in erster Linie auf harten Fakten beruhen, sind mit einem vergleichsweise geringen Realisierungsaufwand verbunden, da sie empirisch erfassbar (deskriptive Informationen wie Alter und Geschlecht) bzw. objektiv messbar (Anzahl der Fehler, Hilfeaufrufe, Reaktionszeiten) sind. Weiche Informationen (Ziele, Pläne, kognitive Prozesse usw.) zu gewinnen und einzusetzen stellt Entwickler meist vor eine anspruchsvollere Aufgabe. Woywod vertritt die Ansicht, dass sie in der Praxis kaum realisierbar sind (Woywod 1997, S. 53). Im Gegensatz zu harten Fakten eröffnen sie einen Interpretationsspielraum, der die Gefahr einer falschen Taxierung in sich birgt.

Bleibt ein Modell über eine Session hinweg konstant, so spricht man von statischen Anwendungen. Verändert es sich während einer Dialogsitzung, so überwiegt der dynamische Charakter. Initiatoren der Modifikationen können sowohl der Benutzer als auch das System sein.

Implizite Modelle beobachten den Anwender und ziehen Rückschlüsse aus seinem Benutzungsverhalten (Usage Modelling). Auf diese Art werden zwar zusätzliche Eingaben vermieden, die Modelle sind jedoch mit großer Unsicherheit behaftet. In Systemen, die Informationen explizit gewinnen, muss der Benutzer Fragen beantworten oder Formulare ausfüllen. Sie sind einfacher zu realisieren und transparenter, da der Anwender weiß, welche persönlichen Informationen verarbeitet werden. Problematisch ist jedoch, dass sie den Benutzer von seinen ursprünglichen Zielen ablenken: Entweder muss er seine Situation ex ante beschreiben oder die präsentierten Informationen ex post bewerten. Erschwerend kommt hinzu, dass aussagekräftige FeedbackMechanismen sehr komplex sein müssen. 
Kann der Benutzer sich die über ihn gespeicherten Daten und/oder deren Interpretationen anzeigen lassen, so spricht man von einem transparenten Modell. Obwohl aus Gründen des Datenschutzes und der Akzeptanz mitunter sinnvoll, sprechen psychologische Aspekte dafür, zumindest manche Rückschlüsse zu verbergen (implizite Modellierung). Dürfte ein Kunde beispielsweise in einem Verkaufsunterstützungssystem seine Taxierung einsehen und darüber hinaus modifizieren, so führten Eitelkeit und terminologische Missverständnisse u. U. zu einer unrealistischen Klassifikation.

Langfristige Benutzermodelle speichern die Merkmale aus einer Dialogsitzung, müssen somit - im Gegensatz zu kurzfristigen - nicht jedes Mal neu aufgebaut bzw. eingegeben werden. Dem Vorteil einer stärkeren Kundenbindung aufgrund des besseren Service stehen rechtliche Restriktionen (s. Kap. 5) und Bedenken der Anwender, persönliche Informationen preiszugeben, gegenüber. Doch selbst wenn es gelingt, langfristige Benutzermodelle in anonymisierter Form zu realisieren, ist $\mathrm{zu}$ beachten, dass sich die Präferenzen der Anwender situationsabhängig bzw. im Zeitablauf verändern können.

Für die Wissensakquisition ist zu klären, ob sich ein System automatisch weiterentwickeln soll, beispielsweise mit Hilfe des maschinellen Lernens, oder ob der Anbieter das abgebildete Wissen und die damit verbundenen Inferenzen personell steuern möchte. So könnte es die Wissensbasis „,verunreinigen“, wenn ein im Internet öffentlich zugängliches Produktberatungssystem von Benutzern lernt, welche die Anwendung nur testen möchten und deshalb kein verlässliches Feedback liefern. Außerdem mag es aus Sicht des Marketing erwünscht sein, die Kontrolle über abgebildete Regeln nicht aus der Hand zu geben.

\section{Bestandsaufnahme der Benutzermodellierung}

Es bot sich an, die Beispiele zur Benutzermodellierung vorwiegend im Internet zu suchen. Die Kapitel 3.1 und 3.2 beschreiben Anwendungen, die bei der Recherche nach Informationen und Produkten im WWW unterstützen. Schulungssysteme, die auf Benutzermodellen basieren, sind ebenfalls schon über das Internet zugänglich (s. Kap. 3.3). Lediglich im Bereich der Mensch-AufgabeZuordnung mussten wir auf klassische Anwendungen zurückgreifen (s. Kap. 3.4)

\subsection{Informationen für Fach- und Führungskräfte}

Unter den lt. Search Engine Watch im August 1998 zehn am meisten frequentierten Suchmaschinen des Internet war keine, die Recherchen in besonderer Weise an die Anforderungen der Benutzer anpasst. Meist lassen sich lediglich die Gewichte einiger Suchparameter und grundlegende Einstellungen, wie die bevorzugte Sprache, beeinflussen. Etwas weiter geht das Programm von Direct Hit: Ähnlich dem Social Filtering reiht das Recherche-System, das u. a. in die 
Suchmaschinen HotBot und Lycos integriert ist, die Treffer nach ihrer Popularität. Ausschlaggebend für die Empfehlung ist, wie oft die präsentierten Links von früheren Benutzern verfolgt wurden. Die Software berücksichtigt aber weder Merkmale des Benutzers, noch liefert sie eine Aussage über die Qualität der Fundstellen.

Ein Trend zur Individualisierung zeichnet sich in Form parametrierbarer Web Portals ab. Es handelt sich dabei um Übersichtsseiten im WWW, auf denen man Links zu verschiedenen Themen findet, die als Ausgangspunkt des regelmäßigen Surfens dienen. In ihrer zweiten Generation können viele der Web Portals an persönliche Präferenzen angepasst werden. So mag der Anwender selbst festlegen, welche Informationen ihm beim Start der Seite automatisch offeriert werden, indem er WWW-Seiten, Usenet- oder Chat-Foren angibt und aus einem Angebot von thematisch untergliederten News-Diensten selektiert. Ein besonderes Ausstattungsmerkmal des Web Portals von Yahoo ist der Pager, der z. B. Aktienkurse beobachtet und bei signifikanten Schwankungen seinen Auftraggeber alarmiert.

\subsection{Verkauf}

Im Verkauf nutzen innovative Systeme wie One-To-One von BroadVision Informationen aus den Geschäftsbeziehungen mit ihren Kunden, um Inhalte zu präsentieren, die zu bereits vorhandenen Produkten passen. Ebenso protokolliert man, welche Links der Benutzer bisher verfolgt hat, um seinen Interessen entsprechende Seiten dynamisch zu generieren oder Werbebanner einzublenden. Den Anwendungen liegen meist regelbasierte Mechanismen, aber auch Künstliche Neuronale Netze (KNN) zugrunde. Beispielsweise merkt sich das KNN von NeuroMatch der Glamus GmbH sowohl das inhaltliche Umfeld, in dem „erfolgreiche“ Werbeflächen standen, als auch die persönlichen Präferenzen der Surfer, um Werbebanner gezielt zu platzieren.

Der Internet-Buchhändler amazon.com verwendet in seinem Recommendation Center das Collaborative Filtering, um für seine Kunden individuelle Leseanregungen zu erzeugen. Als Neukunde wird man aufgefordert, 20 Bücher zu bewerten. Das System findet in seiner Datenbank dann die Kunden mit dem ähnlichsten Geschmack und empfiehlt Literatur, die „verwandten“ Kunden gefällt, von der man aber annimmt, dass sie dem aktuellen Benutzer nicht bekannt ist. Nach demselben Prinzip arbeitet der so genannte „Passport“ des Unternehmens Firefly.

personalogic ist Anbieter eines Werkzeugs für dialogorientierte Beratungssysteme, die - im Gegensatz zum Collaborative Filtering - detaillierte Anforderungsprofile erstellen. In einer Beispiel-Applikation aus der Fahrrad-Branche werden in erster Linie Produkteigenschaften erfragt. Auf höchster Ebene muss der Anwender eingeben, wie viel er für das Fahrrad ausgeben möchte und welche Kategorie er bevorzugt (Mountain Bike, Rennrad usw.). Fehlt dem Kunden das 
technische Know-how, so hilft das System durch problemorientierte Fragen. In weiteren Dialogschritten kann man mit Hilfe von Schiebereglern gewichten, wie wendig oder wie haltbar das Fahrrad sein soll. Das Ergebnis der Beratung ist ein Ranking mit den Optionen, verschiedene Modelle zu vergleichen und genaue Erklärungen der Bewertungen abzurufen.

\subsection{Schulung und Beratung}

Das Lernprogramm Expert System der Temple University erkennt anhand einer Reihe von Fragen, wie viel Lernstoff der Student kennt und wie er am liebsten lernt. Das Programm ist High-School- und College-Studenten über das Internet zugänglich (O. V. 1998c). Die WWW-basierte Teachware AST (Adaptiver Statistik Tutor) der Universität Trier überprüft in einem Test das Statistik-Knowhow der Probanden. Das Kursmaterial wird dann dem aktuellen Wissensstand ebenso angepasst wie verschiedene Hilfen zur Navigation.

AOL verwendet das in Kapitel 3.2 beschriebene Werkzeug von personalogic, um angehende Studenten bei der Auswahl eines geeigneten Colleges zu unterstützen. Die Empfehlung berücksichtigt u. a. regionale Präferenzen, ob man in einem privaten oder einem öffentlichen Institut studieren möchte und wie viel man ausgeben will.

\subsection{Mensch-Aufgabe-Zuordnung}

Workflow-Management-Systeme ordnen einzelne Aufgaben einem Personenkreis zu, indem sie Mitarbeiter und Workflow-Aktivitäten in Rollenkonzepten statisch modellieren. Das WMS BusinessFlow der COI GmbH erweitert diesen Gedanken und stellt so genannte ,dynamische“ Rollen zur Verfügung, mit denen sich komplexe Zuordnungsstrategien von Workflow-Aktivitäten zu Mitarbeitern während der Abwicklung eines Vorgangs realisieren lassen (COI 1998): Workflow-Aktivitäten umfassen dann Attribute, die angeben, welche Qualifikationen ein Mitarbeiter besitzen muss oder sollte, damit er für die Aufgabe in Frage kommt. Für jeden Mitarbeiter kann man festlegen, über welche Fähigkeiten er verfügt. Eine Zuordnungsregel beschreibt, wie das Matching durchgeführt wird, und sollte dabei auch Lastsituationen der einzelnen Mitarbeiter berücksichtigen. Beispielsweise kann man mit dynamischen Rollen die Zuordnungsregel schaffen, bei drohendem Zeitverzug des gesamten Prozesses immer denjenigen Mitarbeiter auszuwählen, der die betreffende Aktivität bereits am häufigsten durchgeführt hat; damit hofft man, den Workflow schneller abzuwickeln. 


\section{Eigene Experimente}

Ein „Blick in die eigene Werkstatt“ skizziert verschiedene Projekte des Bereichs Wirtschaftsinformatik I ${ }^{3}$ der Universität Erlangen-Nürnberg aus Sicht der Benutzermodellierung. Grundlage der Ausführungen ist das Framework aus Kapitel 2.2 .

\subsection{Informationen für Fach- und Führungskräfte}

\subsubsection{Managementinformationen aus dem Internet}

Das Projekt MINT (Managementinformationen aus dem Internet), eine Zusammenarbeit der SAP AG und des FORWISS, erweitert das Executive Information System (EIS) von R/3 um Daten aus dem Internet (Meier 1998). Ein Redaktionsleitstand (Editorial Workbench) erfasst auf der Grundlage von Informationsbedarfen an den Entscheidungsstellen im Unternehmen gezielt relevante Fakten der Unternehmensumwelt und gleicht sie inhaltlich mit internen Werten ab. Die eher qualitativen Informationen aus dem Internet werden dem Benutzer gemeinsam mit den quantitativen Daten aus dem SAP EIS präsentiert.

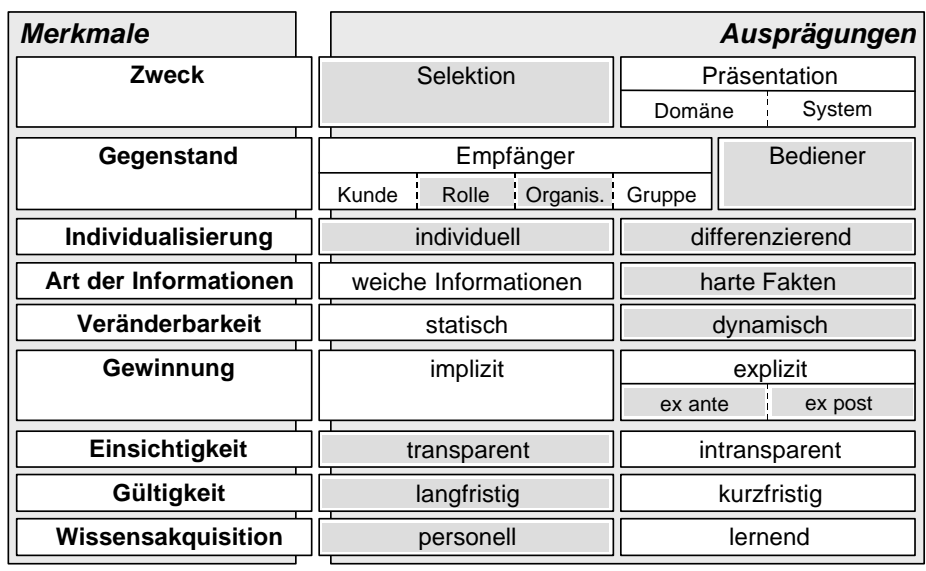

Abbildung 3: Benutzermodell von MINT

3 Dem Bereich Wirtschaftsinformatik I gehören der Lehrstuhl für Betriebswirtschaftslehre, insbesondere Wirtschaftsinformatik I, die Informatik-Forschungsgruppe B (Betriebliche Anwendungen) der Universität Erlangen-Nürnberg sowie die Forschungsgruppe Wirtschaftsinformatik des Bayerischen Forschungszentrums für Wissensbasierte Systeme (FORWISS) an. Diese Einrichtungen werden vom Mitverfasser Mertens in Personalunion geleitet. 
Die Informationsbedarfe im Unternehmen definiert der Anwender im ProfilManager von MINT. Beispielsweise legen Trigger fest, wann Rechercheaufträge aktiviert werden sollen (z. B. periodisch oder signalgesteuert). Der Profil-Manager speichert die Anforderungen in einem hierarchischen Modell, das zwischen Unternehmen, Rollenträger und Benutzer unterscheidet. Während die Benutzerebene persönliche Präferenzen bezüglich der Darstellung und das Vorwissen determiniert, liefert die Rolle des Mitarbeiters Hinweise zu seinen Aufgaben, also seinen objektiven Informationsbedarf. Aus dem Unternehmensprofil lassen sich branchenspezifische Einflussfaktoren gewinnen.

Um Anwender von der personellen Eingabe ihrer Präferenzen in den ProfilManager zu entlasten, soll das System um eine Relevance-Feedback-Funktion erweitert werden. Mit dieser kann der Manager die Fundstellen direkt bewerten, und das System leitet daraus Änderungen des Benutzermodells ab.

\subsubsection{Integration von Controlling- und Marktforschungsdaten in einem Expertisesystem}

Im Rahmen des Kooperationsprojekts INTEX (Integration von Controlling- und Marktforschungsdaten in einem Expertisesystem) zwischen Siemens A\&D (Automation and Drives) AG und FORWISS wurde ein System erstellt, das rasch sich wandelnde Informationsbedarfe von Führungskräften automatisch durch ein adaptives Briefing Book deckt (Cas 1998). Dieses analysiert individuell für jeden Anwender interne Informationen, die aus einem Data Warehouse stammen, und bindet die Ergebnisse zu einem Online-Berichtsbuch zusammen. Die Mitarbeiter können sich damit im Intranet auf wenigen Seiten anhand der wichtigsten Kennzahlen einen Überblick über den Zustand des Unternehmens verschaffen.

\begin{tabular}{|c|c|c|}
\hline Merkmale & & Ausprägungen \\
\hline \multirow[t]{2}{*}{ Zweck } & \multirow[t]{2}{*}{ Selektion } & Präsentation \\
\hline & & Domäne \\
\hline \multirow[t]{2}{*}{ Gegenstand } & Empfänger & \multirow[t]{2}{*}{ Bediener } \\
\hline & Rolle $:$ Organis. & \\
\hline Individualisierung & individuell & differenzierend \\
\hline Art der Informationen & weiche Informationen & harte Fakten \\
\hline Veränderbarkeit & statisch & dynamisch \\
\hline \multirow[t]{2}{*}{ Gewinnung } & \multirow[t]{2}{*}{ implizit } & explizit \\
\hline & & expost \\
\hline Einsichtigkeit & transparent & intransparent \\
\hline Gültigkeit & langfristig & kurzfristig \\
\hline Wissensakquisition & personell & lernend \\
\hline
\end{tabular}

Abbildung 4: Benutzermodell von INTEX 
In einem Journal protokolliert das System alle Anfragen des Benutzers und versucht, zukünftige Bedarfe zu antizipieren. Das Abfragejournal liefert eine genaue Übersicht darüber, welcher Anwender welche Informationen in der Vergangenheit abgerufen hat. Darüber hinaus ergänzen individuelle Einstellungen der Abfragemaske, wie z. B. angezeigte Dimensionen (Kunden, Regionen, Zeiträume), Kennzahlen und Sortierkriterien, das Benutzermodell des Journals.

Um den Inhalt des Briefing Books zu bestimmen, ermittelt man die Wahrscheinlichkeiten zukünftiger Informationsbedarfe. Zur Vorhersage wird das exponentielle Glätten erster Ordnung eingesetzt, da der Bedarf an Abfragen über die Zeit annähernd konstant ist. Potenzielle Anforderungen können anschließend über Nacht berechnet werden und stehen somit am nächsten Tag unmittelbar zur Verfügung. Durch die Vorberechnung sanken die Antwortzeiten der prognostizierten Abfragen von etwa 15 Minuten auf eine Sekunde. Für neue Benutzer eignet sich diese Vorgehensweise nicht, da keine Daten über deren Informationsbedarfe vorliegen. Es ist aber anzunehmen, dass gerade „Novizen“ besonderer Unterstützung bedürfen; das System greift deshalb auf Stereotypen zurück. Anhand von Attributen wie Position, Abteilung und Ausbildung präsentiert das Programm Berichte von Führungskräften mit einem ähnlichen Informationsbedarf.

\subsubsection{Unternehmensreport II}

In Zusammenarbeit mit DATEV entstand das regelbasierte Expertisesystem Unternehmensreport II. Steuerberater erarbeiten damit gutachtenähnliche Berichte an ihre Mandanten, in denen auf der Basis von Buchhaltungs- und Betriebsvergleichsdaten die Erfolgs- Vermögens- und Finanzlage dargestellt ist. Darüber hinaus leitet die Software Diagnosen sowie eventuell auch Therapievorschläge und Prognosen ab.

\begin{tabular}{|c|c|c|c|}
\hline Merkmale & \multicolumn{3}{|c|}{ Ausprägungen } \\
\hline \multirow[t]{2}{*}{ Zweck } & \multirow[t]{2}{*}{ Selektion } & \multicolumn{2}{|c|}{ Präsentation } \\
\hline & & Domäne & System \\
\hline \multirow[t]{2}{*}{ Gegenstand } & \multicolumn{2}{|l|}{ Empfänger } & \multirow[t]{2}{*}{ Bediener } \\
\hline & $\begin{array}{l:l:l}\text { Kunde } & \text { Rolle } & \text { Organis. } \\
\end{array}$ & Gruppe & \\
\hline Individualisierung & individuell & \multicolumn{2}{|c|}{ differenzierend } \\
\hline Art der Informationen & weiche Informationen & \multicolumn{2}{|c|}{ harte Fakten } \\
\hline Veränderbarkeit & statisch & \multicolumn{2}{|c|}{ dynamisch } \\
\hline \multirow[t]{2}{*}{ Gewinnung } & \multirow[t]{2}{*}{ implizit } & \multicolumn{2}{|c|}{ explizit } \\
\hline & & ex ante & ex post \\
\hline Einsichtigkeit & transparent & \multicolumn{2}{|c|}{ intransparent } \\
\hline Gültigkeit & langfristig & \multicolumn{2}{|c|}{ kurzfristig } \\
\hline Wissensakquisition & personell & \multicolumn{2}{|c|}{ lernend } \\
\hline
\end{tabular}

Abbildung 5: Benutzermodell von Unternehmensreport II 
Die Ergebnisse werden in Grafiken, Tabellen und verbalen Passagen (TextGeneration) dokumentiert. Geht man davon aus, die Daten seien analysiert sowie Tabellen und Grafiken bereits erstellt, so liegt die Herausforderung in der „Konfiguration“ der Textpassagen.

Charakteristisch für Expertisesysteme ist ihre Empfängerorientierung. Abbildung 6 deutet an, welche Varianten denkbar sind. Recht schwierig ist es, verschieden lange Versionen der Berichte zu produzieren, denn man kann nicht nur von einem Standardreport aus starten und den „Chefreport“ durch Weglassen von Passagen gewinnen. Einfacher ist die Ableitung einer für den Typ „geduldiger Leser" bestimmten Langversion aus dem Standardreport, indem man eine Lesehilfe in Gestalt eines Glossars hinzufügt.

Persönliche Präferenzen des Empfängers:

Tabellen innerhalb des Reports?

Grafiken integrieren?

Glossar anfügen?
Detaillierungsgrad:

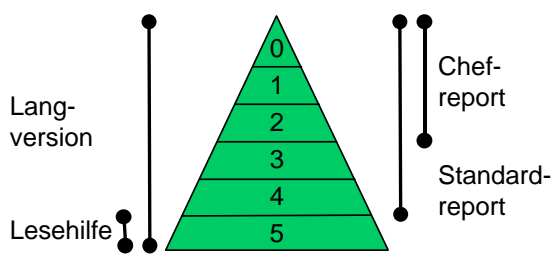

Abbildung 6: Empfängerorientierung

Zwei wichtige Unterscheidungen in Bezug auf die Empfängerorientierung sind:

1. Wünscht der Leser nur eine „,coole“, mehr faktenorientierte Berichterstattung oder auch Wertungen (Kritik)? (Viele Steuerberater scheuen Wertungen, weil sie dadurch ihren Mandanten zu nahe treten könnten.)

2. Sind nur Symptome und Diagnosen erwünscht oder auch Therapievorschläge (Empfehlungen)? Solche Ratschläge aus dem System wirken zuweilen altklug und beleidigen den Empfänger, weil er sich unterschätzt fühlt.

In Unternehmensreport II werden Satzskelette als wertend bzw. empfehlend gekennzeichnet, sodass das System sie auswählen oder weglassen kann. Beispielsweise mag die neutrale Aussage „Vergleicht man den November 1998 mit dem Vorjahresmonat, dann sinkt die Vermögenselastizität von $37 \%$ auf $24 \%$ " um die Wertung „Dies ist sehr negativ zu beurteilen“ ergänzt werden.

\subsubsection{Client-Oriented Branch Reporting and Analysis System}

Marktforschungsinstitute stehen vor der Aufgabe, große Datenmengen für ihre Kunden $\mathrm{zu}$ analysieren und zu visualisieren. Am FORWISS wurde deshalb das Client-Oriented Branch Reporting and Analysis System (COBRAS) entwickelt. Ziel von COBRAS ist es, die Daten des Handelspanels der GfK (Gesellschaft für Konsum-, Markt- und Absatzforschung) zu kundenindividuellen Präsentationen 
aufzubereiten. Zu diesem Zweck hat man die Erfahrungen von Mitarbeitern der GfK in einem regelbasierten System abgebildet. Das Ergebnis von COBRAS sind hochwertige Präsentationscharts, die keiner personellen Nachbearbeitung bedürfen (Christ, in Vorbereitung).

\begin{tabular}{|c|c|c|c|}
\hline Merkmale & \multicolumn{3}{|c|}{ Ausprägungen } \\
\hline \multirow[t]{2}{*}{ Zweck } & \multirow[t]{2}{*}{ Selektion } & \multicolumn{2}{|c|}{ Präsentation } \\
\hline & & Domäne & System \\
\hline \multirow[t]{2}{*}{ Gegenstand } & \multicolumn{2}{|l|}{ Empfänger } & \multirow[t]{2}{*}{ Bediener } \\
\hline & $\begin{array}{l:l}\text { Rolle } & \text { Organis. } \\
\end{array}$ & Gruppe & \\
\hline Individualisierung & individuell & \multicolumn{2}{|c|}{ differenzierend } \\
\hline Art der Informationen & weiche Informationen & \multicolumn{2}{|c|}{ harte Fakten } \\
\hline Veränderbarkeit & statisch & \multicolumn{2}{|c|}{ dynamisch } \\
\hline \multirow[t]{2}{*}{ Gewinnung } & \multirow[t]{2}{*}{ implizit } & \multicolumn{2}{|c|}{ explizit } \\
\hline & & ex ante & ex post \\
\hline Einsichtigkeit & transparent & \multicolumn{2}{|c|}{ intransparent } \\
\hline Gültigkeit & langfristig & \multicolumn{2}{|c|}{ kurzfristig } \\
\hline Wissensakquisition & personell & \multicolumn{2}{|c|}{ lernend } \\
\hline
\end{tabular}

Abbildung 7: Benutzermodell von COBRAS

In einem Repository sind Bausteine bzw. Templates gespeichert, aus denen Charts und Analysen zusammengestellt werden. Welche dieser Elemente in die jeweilige Präsentation einfließen, definieren GfK-Mitarbeiter explizit in Empfängermodellen. Diese enthalten auch kundenindividuelle Layoutvorschriften, wie z. B. Farbschemata, die Sprache der Beschriftungstexte und entsprechende Zahlenformate. Des weiteren kann man dort unternehmensspezifische Kennzahlen beschreiben und Filter, die festlegen, welche Dimensionen den Analysen zugrunde liegen (Warengruppe, Produkt, Zeit usw.). Die Empfängermodelle in COBRAS dienen der Selektion und der Präsentation.

\subsection{Verkauf}

\subsubsection{Infobroker}

Als Zugangssystem zu einer Internet-basierten Auktion für Computer-Hardware benachrichtigt der Infobroker Kunden per E-Mail, wenn Neuigkeiten zu potenziell interessanten Produkten eintreffen (Horstmann 1998). Darüber hinaus erstellt die Software individuelle Newsletters gemäß den Präferenzen der Kunden. Ein weiteres Feature ist der so genannte ,allgemeine Problemzugang“, der in erster Linie Laien unterstützt, die ihre Anforderungen nicht in Form technischer Details spezifizieren können. Infobroker wurde vom FORWISS zusammen mit der AXIS information systems $\mathrm{GmbH}$ entwickelt. 


\begin{tabular}{|c|c|c|}
\hline Merkmale & & Ausprägungen \\
\hline \multirow[t]{2}{*}{ Zweck } & \multirow[t]{2}{*}{ Selektion } & Präsentation \\
\hline & & Domäne \\
\hline \multirow[t]{2}{*}{ Gegenstand } & Empfänger & \multirow[t]{2}{*}{ Bediener } \\
\hline & $\begin{array}{l:l}\text { Rolle } & \text { Organis. } \\
\end{array}$ & \\
\hline Individualisierung & individuell & differenzierend \\
\hline Art der Informationen & weiche Informationen & harte Fakten \\
\hline Veränderbarkeit & statisch & dynamisch \\
\hline \multirow[t]{2}{*}{ Gewinnung } & \multirow[t]{2}{*}{ implizit } & explizit \\
\hline & & ex post \\
\hline Einsichtigkeit & transparent & intransparent \\
\hline Gültigkeit & langfristig & kurzfristig \\
\hline Wissensakquisition & personell & lernend \\
\hline
\end{tabular}

Abbildung 8: Benutzermodell von Infobroker

Den Individualisierungsfunktionen liegen Benutzermodelle zugrunde, die mit Hilfe von Stereotypen (z. B. geschäftlich oder privat als primäre Einsatzfelder) initialisiert werden. Da es sich um einen transparenten Ansatz handelt, mag der Anwender sein Modell auch durch die explizite Auswahl von Interessengebieten anpassen. Infobroker stellt daraufhin Newsletters zusammen, deren Inhalte sich an den jeweiligen Bedürfnissen orientieren. Durch eine Bewertung der einzelnen Informationen mit Hilfe eines direkten Relevance Feedback kann der Kunde sein Benutzermodell verfeinern. Des weiteren zeichnet das System auf, mit welcher Häufigkeit Links des Newsletters verfolgt werden. Stellt Infobroker fest, dass Anwender Verweise auf Inhalte zu einem bestimmten Thema nicht auswählen, so schlägt er vor, diese Kategorie aus dem Benutzerprofil zu streichen.

Der Recommendation Assistant empfiehlt Produkte, die andere Kunden präferierten (Collaborative Filtering). „Ähnlichkeiten“ ermittelt der Recommendation Assistant zum einen über das Kaufverhalten: In einer Datenbank sucht er nach Kunden, deren Produktauswahl der des aktuellen Benutzers möglichst genau entspricht, und schlägt Hardware vor, die diese zusätzlich gekauft haben. Zum anderen orientiert sich der Recommendation Assistant am Benutzungsverhalten, indem er aufzeichnet, welche Links verfolgt wurden, vergleichbare Surfer findet und Seiten aus deren Protokoll empfiehlt, die der aktuelle Kunde noch nicht gesehen hat.

\subsubsection{Electronic Personal Online Shopper}

Der Electronic Personal Online Shopper (EPOS) ist ein elektronischer Einkaufsassistent, dessen Dienste die Kunden einer Mall in Anspruch nehmen können (Griesing, in Vorbereitung). Seine Aufgabe ist es, ihnen regelmäßig wiederkehrende und deshalb meist als lästig empfundene Einkäufe (z. B. Lebensmittel, Körperpflegeartikel, Putzmittel) so weit wie möglich abzunehmen. 
EPOS „merkt" sich beispielsweise, welche Produkte ein Kunde immer wieder kauft (z. B. Butter, Brot, Marmelade, jeweils in einer bestimmten Packungsgröße) und erstellt daraus eine individuelle „Überlebensliste“. Muss der Kunde seinen Vorrat neu auffüllen, so ruft er einfach diese Liste auf, passt sie gegebenenfalls an und schickt sie als Bestellung an die Mall. Für Verbrauchsgüter, die im Haushalt niemals ausgehen dürfen, definiert der Kunde eine Verbrauchsfunktion. Gibt er beispielsweise ein, dass eine 1000g-Packung Müsli ca. zwei Wochen ausreicht, so ordert sein Einkaufsassistent in entsprechenden Abständen bzw. fragt an, ob er nachbestellen soll. Darüber hinaus speichert er das Verfallsdatum von Lebensmitteln, um den Kunden rechtzeitig darauf hinzuweisen, dass dieser ein Produkt aufbrauchen oder nachkaufen sollte.

\begin{tabular}{|c|c|c|c|}
\hline Merkmale & \multicolumn{3}{|c|}{ Ausprägungen } \\
\hline \multirow[t]{2}{*}{ Zweck } & \multirow[t]{2}{*}{ Selektion } & \multicolumn{2}{|c|}{ Präsentation } \\
\hline & & Domäne & System \\
\hline \multirow[t]{2}{*}{ Gegenstand } & \multicolumn{2}{|l|}{ Empfänger } & \multirow[t]{2}{*}{ Bediener } \\
\hline & $\begin{array}{l:l:l}\text { Kunde } & \text { Rolle } & \text { Organis. }\end{array}$ & Gruppe & \\
\hline Individualisierung & individuell & \multicolumn{2}{|c|}{ differenzierend } \\
\hline Art der Informationen & weiche Informationen & \multicolumn{2}{|c|}{ harte Fakten } \\
\hline Veränderbarkeit & statisch & \multicolumn{2}{|c|}{ dynamisch } \\
\hline \multirow[t]{2}{*}{ Gewinnung } & \multirow[t]{2}{*}{ implizit } & \multicolumn{2}{|c|}{ explizit } \\
\hline & & ex ante & ex post \\
\hline Einsichtigkeit & transparent & \multicolumn{2}{|c|}{ intransparent } \\
\hline Gültigkeit & langfristig & \multicolumn{2}{|c|}{ kurzfristig } \\
\hline Wissensakquisition & personell & \multicolumn{2}{|c|}{ lernend } \\
\hline
\end{tabular}

Abbildung 9: Benutzermodell von EPOS

Um seinen Kunden beim Einkauf von Verbrauchsgütern individuell beraten zu können, verfügt EPOS über eine Reihe von Stereotypen, die es ihm erlauben, geeignete Produkte zu empfehlen. So würde etwa der Warenkorb eines Studenten solche Lebensmittel enthalten, die dem Körper die Nährstoffe zuführen, welche dieser bei überwiegend geistiger und sitzender Tätigkeit benötigt (z. B. kalorienarm, aber vitaminreich). Einem Kunden, der schwere körperliche Leistungen erbringen muss (z. B. Sportler, Bauarbeiter), empfiehlt der Einkaufsassistent dagegen kohlehydratreiche Kost mit hohem Brennwert. Daneben verfügt EPOS über einen Fundus an Warenkörben, die auf ganz bestimmte Anlässe zugeschnitten sind (z. B. „Weihnachtsfest“, „Grillfest“; Event Shopping). Je länger der Einkaufsassistent seinen Kunden „kennt“, desto besser passt er dessen Profil an die tatsächlichen individuellen Gegebenheiten an. Ist der Kunde bereit, entsprechende Auskünfte zu geben, so berücksichtigt das System auch so spezielle Eigenschaften wie Diabetes, Allergien oder Übergewicht.

Weitere Stereotypen segmentieren Kunden hinsichtlich ihres Lebensstils (z. B. sportlich, gesellig, gesundheitsbewusst) und ordnen automatisch Warenkörbe zu. 
Darüber hinaus werden Angaben (soweit vorhanden) zum Familienstand (Single oder Familie) oder zum „Kochverhalten“ (koche nie, nur, wenn Besuch kommt, regelmäßig) berücksichtigt. Das Warenkorbprofil wird aufgrund der Einkäufe adaptiert, aber auch der Benutzer selbst kann Änderungen vornehmen.

\subsubsection{Produktberatungskomponente}

Im FORWISS wird versucht, kunden- bzw. benutzerindividuelle Kaufmuster zu entdecken und in der Produktberatung einzusetzen (Timm 1998). Beispielsweise könnte man aus dem Kauf eines hochpreisigen Videorekorders ableiten, dass ein passendes Fernsehgerät aus demselben Preissegment stammen sollte.

Eine Benutzermodellierungskomponente ,,beobachtet“ den Anwender über einen längeren Zeitraum und speichert folgende Merkmale zur Ableitung von Interessen und Präferenzen der Benutzer ab (s. Tabelle 1):

\begin{tabular}{|l|l|}
\hline \multicolumn{1}{|c|}{ Produktmerkmal } & \multicolumn{1}{c|}{ Benutzerinteresse } \\
\hline Preissegment & Ausgabebereitschaft \\
\hline Produktqualität & Qualitätswunsch \\
\hline Produktmarkenimage & Markenbewusstsein \\
\hline Technologie-Level & Technikanspruch \\
\hline Umweltverträglichkeit & Ökologieanspruch \\
\hline$\ldots$ & $\ldots$ \\
\hline
\end{tabular}

Tabelle 1: Transformation von Produktmerkmalen auf Benutzerinteressen

1. Produktkategorie-spezifische Kaufmuster: Der Kauf eines hochpreisigen Fernsehgerätes fließt in den bereits aggregierten Wert, der aus früheren Geschäftsbeziehungen entstanden ist, ein.

2. Anzahl und Volumen der Käufe je Produktkategorie.

3. Kaufhistorie: Um Aspekte wie Markentreue in das Konzept einzubinden, werden Käufe mit den originären Produktdaten protokolliert.

Alternativ besteht für den Nutzer neben dieser impliziten Variante die Möglichkeit, dem Programm seine Interessen und Präferenzen explizit mitzuteilen. Auf Grundlage dieser Informationen passt das Modul die Beratung an den Nutzer an. Ruft ein Kunde beispielsweise das System mit dem Wunsch auf, einen Videorekorder zu erwerben, so initialisieren seine historischen Daten die Anforderung des neuen Kaufs, und das System kann sofort eine individuelle Empfehlung präsentieren. Falls es sich um einen Erstkauf handelt, versucht das Programm, sich an einer übergeordneten Produktkategorie zu orientieren, die eine Aggregation der Merkmalswerte verwandter Güter beinhaltet. So könnten Kaufmuster von Fernsehgeräten bei der Beratung zu Videorekordern berücksichtigt werden. 


\begin{tabular}{|c|c|c|c|}
\hline Merkmale & \multicolumn{3}{|c|}{ Ausprägungen } \\
\hline \multirow[t]{2}{*}{ Zweck } & \multirow[t]{2}{*}{ Selektion } & \multicolumn{2}{|c|}{ Präsentation } \\
\hline & & Domäne & System \\
\hline \multirow[t]{2}{*}{ Gegenstand } & \multicolumn{2}{|l|}{ Empfänger } & \multirow[t]{2}{*}{ Bediener } \\
\hline & $\begin{array}{l:l}\text { Rolle } & \text { Organis. } \\
\end{array}$ & Gruppe & \\
\hline Individualisierung & individuell & \multicolumn{2}{|c|}{ differenzierend } \\
\hline Art der Informationen & weiche Informationen & \multicolumn{2}{|c|}{ harte Fakten } \\
\hline Veränderbarkeit & statisch & \multicolumn{2}{|c|}{ dynamisch } \\
\hline \multirow[t]{2}{*}{ Gewinnung } & \multirow[t]{2}{*}{ implizit } & \multicolumn{2}{|c|}{ explizit } \\
\hline & & ex ante & ex post \\
\hline Einsichtigkeit & transparent & \multicolumn{2}{|c|}{ intransparent } \\
\hline Gültigkeit & langfristig & \multicolumn{2}{|c|}{ kurzfristig } \\
\hline Wissensakquisition & personell & \multicolumn{2}{|c|}{ lernend } \\
\hline
\end{tabular}

Abbildung 10: Benutzermodell der PBK

\subsubsection{Software Consultant}

Der Software Consultant (SCOUT) entstand im Rahmen einer Kooperation des FORWISS mit der DATEV eG (Höhl 1997). Er soll die Kunden des Softwareherstellers, im wesentlichen die Steuerkanzleien, bei der Recherche nach Produkten von DATEV im Internet unterstützen. Da das Unternehmen im Business-to-Business-Bereich agiert, somit dauerhafte Geschäftsbeziehungen zu seinen Kunden unterhält, können umfangreiche Datenbestände zur Selektion und zur Präsentation als langfristige Benutzermodelle abgelegt werden.

\begin{tabular}{|c|c|c|}
\hline Merkmale & & Ausprägungen \\
\hline \multirow[t]{2}{*}{ Zweck } & \multirow[t]{2}{*}{ Selektion } & Präsentation \\
\hline & & Domäne \\
\hline \multirow[t]{2}{*}{ Gegenstand } & Empfänger & \multirow[t]{2}{*}{ Bediener } \\
\hline & Rolle Organis. & \\
\hline Individualisierung & individuell & differenzierend \\
\hline Art der Informationen & weiche Informationen & harte Fakten \\
\hline Veränderbarkeit & statisch & dynamisch \\
\hline \multirow[t]{2}{*}{ Gewinnung } & \multirow[t]{2}{*}{ implizit } & explizit \\
\hline & & ex post \\
\hline Einsichtigkeit & transparent & intransparent \\
\hline Gültigkeit & langfristig & kurzfristig \\
\hline Wissensakquisition & personell & lernend \\
\hline
\end{tabular}

Abbildung 11: Benutzermodell von SCOUT 
Ein News-Modul liefert Informationen, die explizit abonniert wurden. Es berücksichtigt die Rolle des Benutzers, die er in seinem Unternehmen einnimmt, indem es beispielsweise davon ausgeht, dass Systembetreuer eher technische Informationen benötigen, wohingegen sich Führungskräfte stärker für den Nutzen der Produkte interessieren.

Aus den bei DATEV gespeicherten Daten leitet ein Analysemodul erste Hinweise $a b$, wie die Softwareausstattung einer Kanzlei ergänzt werden kann. Wünscht der Kunde detailliertere Empfehlungen, so mag er den regelbasierten Dialog der Produktberatungskomponente durchlaufen. Ein Stereotypenansatz generiert vorab einen individuellen Pfad durch die Problemfelder und belegt sie mit DefaultWerten vor. Der Benutzer darf aber auch die vorgegebene Route verlassen und selbstständig durch die Anforderungsanalyse navigieren.

Während bei der Selektion die Anforderungen der Kanzlei -also eine Organisationsmodellierung - im Vordergrund stehen, liegt der Schwerpunkt bei der Präsentation auf den persönlichen Präferenzen des Benutzers und seiner Rolle innerhalb des Unternehmens.

\subsection{Schulung und Beratung}

\subsubsection{Adaptives Multimediales Präsentationssystem}

Ziel des von FORWISS gemeinsam mit der Forschungsgemeinschaft der Deutschen Keramischen Gesellschaft e.V., Köln entwickelten sowie von der Stiftung Industrieforschung ( $\mathrm{AiF}$ ) geförderten Adaptiven Multimedialen Präsentationssystems (AMPreS) ist es, das rasch anwachsende Wissen auf einem stark erklärungsbedürftigen Themengebiet interessant und verständlich einer heterogenen Zielgruppe multimedial zu präsentieren. In diesem Branchenkatalog für technische Keramik dient das Benutzermodell besonders der Informationsfilterung, um individuelle Touren durch die Domäne anzubieten. Dem Benutzer ist es dabei freigestellt, ob er sich führen lässt oder ob er selbst durch den Katalog navigiert (Rössel 1998).

Um unterschiedlichen Motivationen, Kenntnissen und Fähigkeiten von potenziellen Anwendern, wie z. B. Technikern, Kaufleuten, Lehrern, Journalisten, Studenten und Schülern, gerecht zu werden, ordnet AMPreS seine Benutzer in die in Tabelle 2 dargestellten Dimensionen ein. Neben den stereotypischen Ausprägungen hält das System auch individuelle Werte, z. B. platzierte Lesezeichen oder gesuchte Termini, fest.

Für Benutzer, die das System nicht kennen, ist eine spezielle Einführung implementiert. Die wenige Bildschirmseiten umfassende Startroutine informiert über den Inhalt und die Funktionsweise der Software, gewinnt aber auch erste Erkenntnisse über den Anwender: In kurzen Textpassagen sind unterschiedliche Termini enthalten, die ein Laie vermutlich nicht kennt, ein Fachmann oder ein Experte aber beherrschen sollte; Abrufe von integrierten Zusatzerklärungen wer- 
den als Indiz für fehlendes Fachwissen gewertet. Nachdem der Benutzer die Einführung durchlaufen hat, enthält sein Benutzermodell zunächst nur einige stereotypische Werte.

\begin{tabular}{|l|l|l|l|l|}
\hline \multicolumn{1}{|c|}{ Dimensionen } & \multicolumn{3}{c|}{ Ausprägungen } \\
\hline Ziel & Überblick & Details & \\
\hline Zeitbudget & wenig & mittel & viel \\
\hline Fachwissen & Laie & Fachmann & Experte \\
\hline Systemwissen & Anfänger & Routinier & \multicolumn{1}{|c|}{} \\
\hline Kognitiver Stil & Holist & Serialist & & \\
\hline Präsentationstyp & intellektuell & fotografisch & cineastisch & auditiv \\
\hline
\end{tabular}

Tabelle 2: Dimensionen des Benutzermodells

Die Tourkomponente, der so genannte „Intelligent Tour Guide“, selektiert aus der Datenbasis interessante und passende Medien und legt anschließend eine sinnvolle Präsentationsfolge fest. Sie beschränkt sich nicht auf eine Art der Führung, sondern differenziert Touren, beispielsweise solche, die sich an pädagogischen Zielen orientieren. Das System reagiert auf Veränderungen der individuellen Merkmale und Wünsche, indem es vollkommen neue Führungen zusammenstellt. Diese besondere Form der individuellen Benutzerunterstützung wurde vor allem für ungeübte Anwender entwickelt, die sich so auf den Inhalt konzentrieren können und nicht überlegen müssen, wie sie zu passenden Informationen gelangen.

\begin{tabular}{|c|c|c|c|}
\hline Merkmale & \multicolumn{3}{|c|}{ Ausprägungen } \\
\hline \multirow[t]{2}{*}{ Zweck } & \multirow[t]{2}{*}{ Selektion } & \multicolumn{2}{|c|}{ Präsentation } \\
\hline & & Domäne & System \\
\hline \multirow[t]{2}{*}{ Gegenstand } & \multicolumn{2}{|l|}{ Empfänger } & \multirow[t]{2}{*}{ Bediener } \\
\hline & $\begin{array}{l:l}\text { Rolle } & \text { Organis. } \\
\end{array}$ & Gruppe & \\
\hline Individualisierung & individuell & \multicolumn{2}{|c|}{ differenzierend } \\
\hline Art der Informationen & weiche Informationen & \multicolumn{2}{|c|}{ harte Fakten } \\
\hline Veränderbarkeit & statisch & \multicolumn{2}{|c|}{ dynamisch } \\
\hline \multirow[t]{2}{*}{ Gewinnung } & \multirow[t]{2}{*}{ implizit } & \multicolumn{2}{|c|}{ explizit } \\
\hline & & ex ante & ex post \\
\hline Einsichtigkeit & transparent & \multicolumn{2}{|c|}{ intransparent } \\
\hline Gültigkeit & langfristig & \multicolumn{2}{|c|}{ kurzfristig } \\
\hline Wissensakquisition & personell & \multicolumn{2}{|c|}{ lernend } \\
\hline
\end{tabular}

Abbildung 12: Benutzermodell von AMPreS 


\subsubsection{Stadtinformationssystem}

Ein Tourist in der Region Nürnberg, Fürth und Erlangen steht meist vor dem Problem, dass er sich einer unüberschaubaren Auswahl an interessanten Sehenswürdigkeiten und anderen Freizeitaktivitäten gegenüber sieht. In einem von der Deutschen Forschungsgemeinschaft (DFG) geförderten Projekt am FORWISS wird ein Internet-basiertes Beratungssystem konzipiert und entwickelt, das bei der persönlichen Freizeitplanung unterstützt (Schuhbauer 1998).

\begin{tabular}{|c|c|c|}
\hline Merkmale & & Ausprägungen \\
\hline \multirow[t]{2}{*}{ Zweck } & \multirow[t]{2}{*}{ Selektion } & Präsentation \\
\hline & & Domäne \\
\hline \multirow[t]{2}{*}{ Gegenstand } & Empfänger & \multirow[t]{2}{*}{ Bediener } \\
\hline & Rolle $:$ Organis. & \\
\hline Individualisierung & individuell & differenzierend \\
\hline Art der Informationen & weiche Informationen & harte Fakten \\
\hline Veränderbarkeit & statisch & dynamisch \\
\hline \multirow[t]{2}{*}{ Gewinnung } & \multirow[t]{2}{*}{ implizit } & explizit \\
\hline & & ex post \\
\hline Einsichtigkeit & transparent & intransparent \\
\hline Gültigkeit & langfristig & kurzfristig \\
\hline Wissensakquisition & personell & lernend \\
\hline
\end{tabular}

Abbildung 13: Benutzermodell des Stadtinformationssystems

Das Stadtinformationssystem generiert einen individuellen Plan für den Aufenthalt. Nach möglichst wenigen Eingaben erstellt es aus einzelnen Bausteinen einen kompletten Freizeitvorschlag. Dabei werden sowohl zeitliche Restriktionen als auch individuelle Präferenzen berücksichtigt: Über Stereotypen ermittelt das System ein Profil und empfiehlt Freizeitaktivitäten im angegebenen Zeitraum. Ist der Benutzer nicht damit einverstanden, so hat er die Möglichkeit, sein Profil zu ändern.

Die Merkmale der Stereotypen entsprechen den Kategorien der Freizeitobjekte: Für jede Rubrik (z. B. Theater), alternativ für jede Oberkategorie (z. B. Kultur), bildet ein Stereotyp das Interesse eines ,typischen“ Nutzers ab. Da die Freizeitbranche ausgesprochen dynamisch ist, verfolgt eine Lernkomponente Trends und schreibt die Profile der Anwender fort.

Oftmals plant ein Tourist nicht nur für sich selbst, sondern für eine Gruppe von Personen. Um Zielkonflikte aufzulösen, die sich aus den mitunter divergierenden Präferenzen der einzelnen Mitglieder ergeben, werden Einzelstereotypen zu Gruppenprofilen verdichtet. Dabei ist zu beachten, dass die Interessen der Beteiligten (z. B. von Kindern und schwangeren Frauen) unterschiedlich gewichtet werden müssen. Die Benutzermodelle des Stadtinformationssystems können 
dialogorientiert, durch Beobachtung des Anwenderverhaltens und im spielerischen Ablauf erhoben werden. Um die Geduld des Anwender nicht übermäßig zu strapazieren, sollte das Programm möglichst wenige und unkritische Fragen stellen; wenn der Kunde möchte, kann er auch ein individuelles Profil definieren. Sofern gewünscht, speichert die Anwendung das Interessenprofil, um in späteren Sitzungen redundante Eingaben zu vermeiden und bereits gesehene „Attraktionen" zu berücksichtigen.

Die abgelegten Profile bündelt das Stadtinformationssystem im Sinne des Collaborative Filtering zu Interessengemeinschaften, so genannten „Cliquen“. Ein Algorithmus untersucht, welche Freizeitaktivitäten bei welcher Clique besonders beliebt waren (Relevance Feedback), und lässt die Ergebnisse in neue Pläne einfließen.

\section{Hindernisse und Gefahren}

Bedenken der Anwender rühren in erster Linie aus der potenziellen Verletzung ihrer Persönlichkeitsrechte. Negativbeispiele im Internet senken die Bereitschaft, persönliche Informationen bekanntzugeben: So soll die Version 4.5 des NetscapeBrowsers Bookmarks und Adressbücher an Netscape übertragen, damit das Unternehmen auf seiner Web-Site Netcenter eine individualisierte Arbeitsumgebung anbieten kann (O. V. 1998b). Die Internet-Werbe-Company DoubleClick erforscht mit Hilfe der Cookie-Technologie das Verhalten der Surfer über mehrere Sites hinweg und speichert die Informationen in Adressdatenbanken.

Das Informations- und Kommunikationsdienste-Gesetz (IuKDG) und das Bundesdatenschutzgesetz (BDSG) regeln in Deutschland die rechtlichen Aspekte der Benutzermodellierung im Internet. Artikel 2 des IuKDG, das Teledienstedatenschutzgesetz (TDDSG), beschreibt den Schutz personenbezogener Daten. Darunter fallen die in diesem Aufsatz skizzierten Online-Ansätze zur Benutzermodellierung. Grundsätzlich gilt die Maxime, „,keine oder so wenige personenbezogene Daten wie möglich zu erheben, zu verarbeiten und zu nutzen“ (§ 3 IV TDDSG). Darüber hinaus muss der Anwender explizit einwilligen, sofern ein Unternehmen plant, Bestandsdaten für Werbung, Marktforschung oder die bedarfsgerechte Gestaltung technischer Einrichtungen zu verwenden (§ 5 II TDDSG).

So stellt sich die Frage, ob es nicht „Netsurfer“ gibt, die bereit wären, ihre Daten relativ detailliert aufzeichnen zu lassen, wenn sie im Gegenzug nur Informationen im weitesten Sinne erhielten, die auf ihre Interessen und Bedürfnisse zugeschnitten sind, wenn sie also von sie nicht interessierenden Produktinformationen und Werbemaßnahmen verschont blieben (Mertens 1996, S. 524). Wenn man über die weitere Diffusion der Idee nachdenkt, stößt man auf überraschende Hindernisse. Beispielsweise sind Anbieter von Suchmaschinen nicht unbedingt daran interessiert, die Recherche sehr zielgerichtet und auf dem 
kürzesten Weg zu organisieren. Vielmehr profitieren Sie auch davon, dass der Benutzer vorübergehend ungewollt Umwege einschlägt, weil er auf den zusätzlichen Web-Seiten zusätzliche Werbebanner sieht, was wiederum den Umsatz des Suchmaschinenanbieters fördert.

Im Marketing versucht man, Informationen so stark zu filtern und an den potenziellen Bedürfnissen der Kunden auszurichten, dass nur noch wenige, aber - wie man hofft - wirkungsvolle Werbebotschaften beim Konsumenten ankommen. Hier könnte man allerdings auch eine Gefahr sehen, die schon in der Diskussion um die automatische oder teilautomatische Zuweisung von Arbeitern zu Arbeitsplätzen und umgekehrt, also beim so genannten Work-AssignmentProblem, eine Rolle gespielt hat: die Gefahr, dass Profile an den Rändern zu scharf sind und ungerechtfertigt fortgeschrieben werden. So habe beispielsweise ein Liebhaber von Operetten bisher die Beschäftigung mit Opern weit von sich gewiesen mit dem Argument, dass ihm diese als zu schwere Unterhaltung erschienen. Man würde diesem Konsumenten also immer nur Operettenmusik anbieten. Vielleicht verlässt er aber doch gerne seinen „Geschmackskäfig“, wenn man ihm erstmals Opernmusik zu Gehör bringt, die dem Operetten-Stil ähnlich ist, beispielsweise von Puccini oder Lortzing, und möglicherweise kommt er auf diesem Wege schlussendlich sogar über Verdi zu Wagner und damit weit weg von der Operette (Mertens 1996).

\section{Resümee und Ausblick}

Es bleibt festzuhalten: Trotz der langen Geschichte der Benutzermodellierung in der Informatik und in der Wirtschaftsinformatik ist das, was wir in der Wirklichkeit vorfinden, bescheiden. Wir haben aber keinen Zweifel, dass wir den eingeschlagenen Weg weiterverfolgen müssen, denn wissenschaftlicher und fachlicher Fortschritt lebt auch von der Wechselwirkung zwischen Typisierung und Differenzierung. Und sollten wir irgendwann die Vorkenntnisse und Präferenzen der Benutzer, Adressaten usw. so, wie wir sie hier verstanden haben, abbilden können, dann bleibt uns als Herausforderung, Vorarbeiten von informatikorientierten Psychologen zur Abbildung der Psyche in Informationssystemen (vgl. Dörner 1998) für die betriebliche IV zu verwenden.

\section{Literaturverzeichnis}

Ackerman, M. (1994): Answer Garden: A Tool for Growing Organizational Memory. Dissertation, Massachusetts 1994. 
Becker, M./Vogler, P./Österle, H. (1998): Workflow-Management in betrieblicher Standardsoftware. WIRTSCHAFTSINFORMATIK 40 (1998) 4, S. 318-328.

Bodendorf, F. (1992): Benutzermodelle - ein konzeptioneller Überblick. WIRTSCHAFTSINFORMATIK 34 (1992) 2, S. 233-245.

Cas, K./Bingler, D. (1998): Adaptive Briefing Books mit Hilfe einer Client-Server-Architektur mit integriertem Abfragejournal. In: Timm, U./Rössel, M. (Hrsg.): ABIS-98: 6. Workshop Adaptivität und Benutzermodellierung in interaktiven Softwaresystemen. FORWISS-Report FR-1998-004, Erlangen 1998, S. 1-6.

Christ, V. (in Vorbereitung): COBRAS - Ein Beitrag zur Analyse und Visualisierung von Marktforschungsergebnissen. Dissertation, Nürnberg, in Vorbereitung.

COI (1998): Online-Systemhandbuch COI-BusinessFlow 3.5. Herzogenaurach 1998.

Dörner, D. (1998): Künstliche Seelen. aviso o. Jg. (1998) 2, S. 34-42.

Griesing, J. (in Vorbereitung): Konzeption und prototypische Realisierung eines Einkaufsassistenten für die Kunden einer Electronic Shopping Mall. Diplomarbeit, Nürnberg, in Vorbereitung.

Höhl, M./Ponader, M. (1997): Knowledge Based Marketing - Individuelles Softwaremarketing über das World Wide Web. In: Link, J. u. a. (Hrsg.): Handbuch Database Marketing, Ettlingen 1997, S. 583-598.

Horstmann, R./Timm, U./Mertens, P. (1998): Concept and Realization of an Internet-based Infobroker for Hardware Components. In: Doukidis, J. u. a. (Hrsg.): Proceedings of the 11th International Bled Electronic Commerce Conference: Electronic Commerce in the Information Society. Kranj 1998, S. 505-517.

Kobsa, A. (1985): Benutzermodellierung in Dialogsystemen. Berlin u. a. 1985.

Kotler, Ph. (1997): Managing Direct and Online Marketing. In: Link, J. u. a. (Hrsg.): Handbuch Database Marketing. Ettlingen 1997, S. 491-511.

Langer, K. (1995): Flexible Hypermedia-Systeme in der computerunterstützten Aus- und Weiterbildung. Wiesbaden 1995.

Link, J./Hildebrand, V. (1993): Database Marketing und Computer Aided Selling. München 1993.

Meier, M. (1998): Integration von Informationen aus dem Internet in das SAPEIS: Konzeption und prototypische Realisierung eines Redaktions-Leitstands. In: Uhr, W./Breuer, S.-E. (Hrsg.): Integration externer Informationen in Management Support Systems. Dresden 1998, S. 105-122.

Mertens, P. (1995a): Wirtschaftsinformatik - Von den Moden zum Trend. In: König, W. (Hrsg.): Wirtschaftsinformatik '95. Heidelberg 1995, S. 2564. 
Mertens, P. (1995b): Mass Customization (Massen-Maßfertigung). WIRTSCHAFTSINFORMATIK 37 (1995) 5, S. 503-505.

Mertens, P./Schumann, P. (1996): Electronic Shopping - Überblick, Entwicklungen und Strategie. WIRTSCHAFTSINFORMATIK 38 (1996) 5, S. 515530.

Mertens, P. (1997): Für Sie gelesen: Resnick, B./Varian, H. R., Recommender Systems. WIRTSCHAFTSINFORMATIK 39 (1997) 4, S. 401-404.

Negroponte, N. (1995): Total Digital. München 1995.

Oard, D. (1997): The State of the Art in Text Filtering. User Modeling and UserAdapted Interaction 7 (1997) 3, S. 141-178.

Oberweis, A. (1996): An Integrated Approach for the Specification of Processes and Related Complex Structured Objects in Business Applications. Decision Support Systems 17 (1996) 1, S. 31-53.

O. V. (1998a): Der Euro bringt den Durchbruch beim Elektronischen Handel. Frankfurter Allgemeine Zeitung vom 1998-04-04, S. 14.

O. V. (1998b): Netscape dringt in Privatsphäre ein. Computer Zeitung 29 (1998) 33, S. 9.

O. V. (1998c): Lernprogramme stellen sich individuell auf Studenten ein. Bild der Wissenschaft Online, Meldung vom 1998-03-03, http://www. wissenschaft.de/bdw/ticker, abgerufen am 1998-04-03.

Peppers, D./Rogers, M. (1993): The One to One Future. New York u. a. 1993.

Resnick, P./Varian, H. R. (1997): Recommender Systems. Communications of the ACM 40 (1997) 3, S. 56-58.

Rössel, M. (1998): Elektronische Branchenkataloge - Nutzungsmöglichkeiten und Akzeptanzförderung. In: Hippner, H./Meyer, M./Wilde, K. D. (Hrsg.): Computer-based Marketing - Das Handbuch zur Marketinginformatik. Braunschweig, Wiesbaden 1998, S. 141-148.

Schuhbauer, H. (1998): Individualisierung von Angeboten in einem Internet-basierten Stadtinformationssystem. In: Wilke, W., Schumacher, J. (Hrsg.): KI-98 Workshop on Intelligent Systems and Electronic Commerce. Kaiserslautern 1998, S. 31-40.

Timm, U./Rosewitz, M. (1998): Benutzermodellierung in der Elektronischen Produktberatung - Konzept und prototypische Realisierung in einer OnlineUmgebung. In: Timm, U./Rössel, M. (Hrsg.): ABIS-98: 6. Workshop Adaptivität und Benutzermodellierung in interaktiven Softwaresystemen. FORWISS-Report FR-1998-004, Erlangen 1998, S.105-112.

Wahlster, W./Kobsa, A. (1989): User Models in Dialog Systems. In: Kobsa, A./Wahlster, W. (Hrsg.): User Models in Dialog Systems. Berlin 1989, S. 4-34. 
Woywod, A. (1997): Verfeinerung von Expertisesystemen durch Benutzermodellierung. Frankfurt am Main u. a. 1997. 